\title{
Bioadhesive vaginal tablets containing spray dried microspheres loaded with clotrimazole for treatment of vaginal Candidiasis
}

\author{
NARESH VISHAL GUPTA* \\ SHIRODKER NATASHA \\ ANIL GETYALA \\ RAMNATH SUDEENDRA BHAT \\ Department of Pharmaceutics \\ JSS College of Pharmacy, JSS University \\ Sri Shivarathreeshwara Nagar \\ Mysore-570015, Karnataka, India
}

Accepted May 7, 2013

\begin{abstract}
The aim of the present investigation was to prepare and evaluate novel bioadhesive vaginal tablets containing clotrimazole loaded microspheres in order to provide long-term therapeutic activity at the site of infection. Tablets were prepared by incorporating drug loaded microspheres and using bioadhesive polymers hydroxypropylmethylcellulose, sodium carboxymethylcellulose and Carbopol. Microspheres were prepared by the spray drying technique using Eudragit RS-100 and Eudragit RL-100. Microspheres were characterized by SEM, DSC, FTIR, particle size analysis and evaluated for percentage yield, drug loading, encapsulation efficiency and in vitro drug release. To achieve bioadhesion to the mucosal tissue, optimized microspheres were incorporated into bioadhesive tablets and were evaluated for in vitro drug release, in vitro and in vivo mucoadhesion. FTIR and DSC studies showed that no chemical interaction occurred between the drug and polymers. The sphericity factor indicated that the prepared microspheres were spherical. Formulation Mt6 indicated a controlled in vitro drug release and good bioadhesive strength. The in vivo images confirmed the bioadhesion and retention property of tablets up to $24 \mathrm{~h}$. The results indicated that this drug delivery system can be explored for controlled intravaginal drug release.
\end{abstract}

Keywords: clotrimazole, microspheres, bioadhesive vaginal tablet

Conventional vaginal formulations are associated with disadvantages of low retention in the vaginal epithelium, leakage and messiness, thereby causing inconvenience to the user. To circumvent these problems, bioadhesive drug delivery systems are propagated (1). Bioadhesive vaginal tablet formulations capable of delivering the active agent

* Correspondence; e-mail: vkguptajss@gmail.com 
for an extended period of time at a predictable rate have been developed and studied recently (2). Mucoadhesive drug delivery systems offer several advantages such as localization at a given target site, prolonged residence time at the site of drug absorption, and intensified contact with the mucosa increasing the drug concentration gradient. Hence, uptake and consequently bioavailability of the drug may be increased, frequency of dosing reduced and patient compliance improved (3). Among the various routes, the vaginal and rectal lumen have also been explored for the delivery of active agents both systemically and locally. Active agents intended for systemic delivery by this route of administration bypass the hepatic first-pass metabolism. Delivery systems quite often suffer from migration within the vaginal/rectal lumen, which might affect the delivery of the active agent to the specific location. Use of mucoadhesive polymers for the development of delivery systems helps in reducing their migration, thereby promoting better therapeutic efficacy. Various mucoadhesive polymers like poly(acrylates), chitosan, cellulose derivatives (hydroxyethylcellulose, hydroxypropylcellulose and hydroxypropylmethylcellulose), hyaluronic acid derivatives, pectin, tragacanth, starch, poly(ethylene glycol), sulfated polysaccharides, carrageenean, Na-alginate and gelatine have been used in vaginal drug delivery (4).

Imidazole derivative clotrimazole (CTZ) is a broad-spectrum antimycotic agent effective against pathogenic dermatophytes, yeasts and several species of Candida, Trichophyton, Microsporum, Epidermophyton and Malassezia. However, clotrimazole is known to be very effective locally and causes no major side effects.

Conventional drug delivery systems (exemplified by tablets, creams, gels, pessaries, and foams) suffer from poor retention in the vaginal tract, since they are removed in a short time by the tract's self-cleansing action due to vaginal fluids (5). Multiple daily doses of conventional formulations result in poor patient compliance. To overcome such problems, delivery systems with bioadhesive polymers that prolong drug action on the vaginal mucosa were developed (6-8). A thorough literature search revealed a lack of information on combinations of HPMC, SCMC and Carbopol based bioadhesive vaginal tablets for controlled drug release containing clotrimazole loaded microspheres. Development of a controlled release delivery system for clotrimazole would provide long-term therapeutic concentrations at the site of infection by a single dose, leading to eradication of the infection (8).

The main goal of this research was to design and evaluate a vaginal delivery system for the local treatment of vaginal Candidiasis.

\section{EXPERIMENTAL}

\section{Materials}

Clotrimazole was a gift sample from KAPL, India. Eudragits RS 100 and RL 100 were obtained from Vikram Thermo Ltd, India. Dichloromethane, hydroxypropyl-methylcellulose (HPMC) and sodium carboxymethylcellulose (SCMC) were purchased from Loba Chemie Pvt Ltd., India. Carbopol 934 was a gift sample from Micro labs, India. All other reagents used in the study were of analytical grade. 
N. Vishal Gupta et al.: Bioadhesive vaginal tablets containing spray dried microspheres loaded with clotrimazole for treatment of vaginal Candidiasis, Acta Pharm. 63 (2013) 359-372

\section{Preparation of microspheres by spray drying}

Weighted amounts of Eudragit RS-100 or Eudragit RL-100 (as release controlling agents) were dissolved in dichloromethane. To this solution, a required amount of CTZ was added. Spray drying was co-currently performed using a LSD-48 mini spray drier (JISL, India) with a standard $0.5 \mathrm{~mm}$ nozzle. Inlet temperature was maintained at $80{ }^{\circ} \mathrm{C}$, flow rate was maintained at 5-6 $\mathrm{mL} \mathrm{min}^{-1}$, compressed spray air flow was maintained at $10 \mathrm{~L} \mathrm{~min}^{-1}$ and air pressure was kept at $1.47 \times 10^{5} \mathrm{~N} \mathrm{~m}^{-2}$. Solvent evaporation by a flow of heated air aspirated by a pump (29-35 ASP, corresponding to 50-60 $\mathrm{mm}$ of $\mathrm{H}_{2} \mathrm{O}$ negative pressure) induced the formation of solid microspheres from the drop. The obtained microspheres were separated in a cyclone separator and settled into a collector (9). Formulation chart of microspheres is given in Table I. The spray drying method of microencapsulation involves dispersion of a solution containing polymer and drug with an additional medium in which the drug and polymer cannot dissolve. Additional medium is required to precipitate the microspheres.

\section{Micromeritic properties}

Angle of repose is the maximum angle possible between the surface of a pile of microspheres and the horizontal plane. Fixed funnel method was employed (10).

Apparent bulk density was determined by pouring the samples in bulk into a graduated cylinder. Tapped density was determined by placing a graduated cylinder containing a known mass of powder on a mechanical tapper apparatus (ETD-1020, Electrolab, India). Samples were tapped until no further reduction in volume of the sample was observed. Carr's index was calculated (10).

\section{Compatibility studies}

Fourier transform infrared spectroscopy (FTIR). - Potassium bromide was mixed with the sample, compressed into a pellet and spectra were taken between $400-4000 \mathrm{~cm}^{-1}$ using an FTIR spectrophotometer (FT-IR-8400-S, Shimadzu, Japan).

Differential scanning calorimetry (DSC). - About $2 \mathrm{mg}$ of sample was placed in an aluminium pan and hermetically sealed. DSC thermograms were recorded from 0 to $200{ }^{\circ} \mathrm{C}$. The instrument was calibrated using high purity indium metal as standard. Dynamic scans were taken in a nitrogen atmosphere at the heating rate of $5{ }^{\circ} \mathrm{C} \mathrm{min}-1$ (DSC-60 Shi$\operatorname{madzu}$ ).

\section{Particle size analysis and sphericity of microspheres}

Particle size analysis of microspheres was carried out by optical microscopy. About 200 microspheres were selected randomly and their size was determined using an optical microscope (SK-6, Suswox Optic, India) fitted with a standard micrometer scale.

To determine sphericity, tracings of CTZ microspheres (magnification $45 \times$ ) were taken on black paper using a camera lucida (Model Prism type, Rolex, India). Sphericity factor $(S)$ was calculated as: 


$$
S=\frac{P^{2}}{12.56 \times A}
$$

where $A$ is the area $\left(\mathrm{cm}^{2}\right)$ and $P$ is the perimeter $(\mathrm{cm})$ of the circular tracing (11).

Scanning electron microscopy (SEM) photographs were taken using a scanning electron microscope (Joel-LV-5600, Japan) at room temperature. Samples were fixed on a scanning electron microscope sample holder with a double-sided adhesive tape and coated with a layer of gold of $1.5 \times 10^{-10} \mathrm{~m}$ for 2 min using a sputter coater (Edwards 3-150 $\AA$, England) in a vacuum of $30.4 \mathrm{kPa}$ of argon gas. Photographs were observed for morphological characteristics and to confirm the spherical nature of microspheres.

\section{Drug loading and encapsulation efficiency}

Microspheres $(100 \mathrm{mg}$ ) were extracted with $5 \mathrm{~mL}$ of methanol, diluted with $\mathrm{pH} 4.5$ citrate-phosphate buffer, filtered and analyzed for drug content after suitable dilution, at $264 \mathrm{~nm}(12)$.

\section{Tablet preparation}

Incorporation of microspheres in a tablet was carried out in the following way. Optimized microspheres equivalent to $100 \mathrm{mg}$ of clotrimazole along with HPMC, SCMC, Carbopol, magnesium stearate and calcium hydrogenphosphate were physically mixed and were punched into tablet by direct compression using a tablet punching machine (Rimek Minipress, India) (Table II) (13). Instead of microspheres, $100 \mathrm{mg}$ of pure clotrimazole was used to get a control tablet.

\section{Evaluation of tablets}

Hardness of core tablets was measured using an Erweka tablet hardness tester (IHT 100, Erweka, India). Ten tablets were tested for each batch. Ten tablets were randomly selected and the average mass and standard deviation were calculated. For thickness measurements, ten tablets were randomly selected from each batch and measured by a micrometer screw gauge (293-821, Mitutoyo, Japan). Ten tablets were weighed and placed in the Roche friabilator apparatus (EF-2, Electrolab, India) and rotated at $25 \mathrm{rpm}$ for 4 minutes. Afterwards, the tablets were dedusted and weighed again. Percentage friability was calculated (14).

Drug content. - Ten tablets of each formulation were crushed in a mortar. A quantity of powder equivalent to $100 \mathrm{mg}$ of CTZ was taken, transferred to a $100-\mathrm{mL}$ volumetric flask and diluted with $\mathrm{pH} 4.5$ citrate phosphate buffer (15). Absorbance was measured at $264 \mathrm{~nm}$.

Swelling. - Each tablet was weighed and then placed in $600 \mathrm{~mL}$ citrate-phosphate buffer $\mathrm{pH} 4.5$ in a basket at $37 \pm 0.5^{\circ} \mathrm{C}$. The samples were periodically weighed after removing the excess water on the surface with a filter paper (2). 


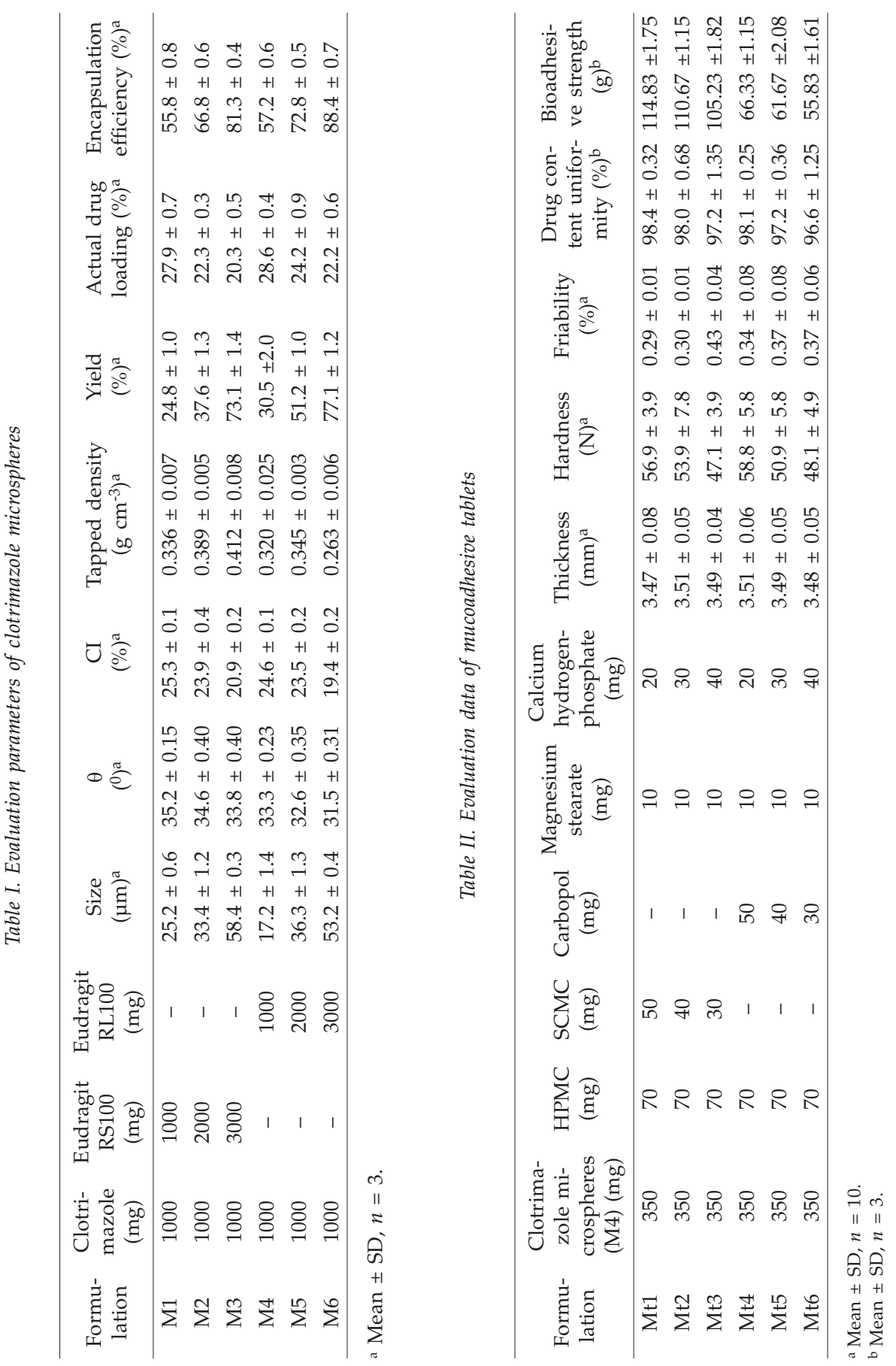


In vitro mucoadhesive strength. - Sheep vaginal mucosa was used as a model membrane. The mucoadhesive potential of each tablet formulation was determined by measuring the force required to detach the formulation from the vaginal mucosal tissue. A section of sheep vaginal mucosa was fixed on each of the two glass slides using cyanoacrylate adhesive. The formulation was placed on the first slide while another slide with mucosal section attached to a pan was placed in inverted position above the first slide. Both slides with the formulation between them were held in contact for 5 min using a preload of $50 \mathrm{~g}$ to ensure intimate contact between them. Weight was then kept rising in the pan until the mucosa on each slide got detached from each other. The weight required to detach the tablet from vaginal mucosa was noted as bioadhesive strength (16).

\section{In vitro drug release studies}

Release studies were carried out on all formulations in triplicate, employing a basket type dissolution tester-USP XXII (TDT-08L, Electrolab, India) using $600 \mathrm{~mL}$ of pH 4.5 citrate phosphate buffer as dissolution medium at $100 \mathrm{rpm}$ at $37 \pm 0.5{ }^{\circ} \mathrm{C}$ to mimic the vaginal conditions (15). Five $\mathrm{mL}$ of the sample was withdrawn at different intervals and analyzed by the UV method at $264 \mathrm{~nm}(2,7,17)$.

\section{In vitro antifungal studies}

Antifungal activity was evaluated by the cup-plate method using agar plates inoculated with Candida albicans. A volume of $20 \mathrm{~mL}$ of sterilized agar media was dispersed into a sterilized Petri dish and allowed to solidify. Each Petri dish was divided into two sectors, and a bore $(6 \mathrm{~mm})$ was made in each sector using a sterile cork borer. Each bore in a different sector was loaded with a placebo tablet (negative control), metronidazole pure drug (positive control) and a microspheres loaded CTZ tablet. Petri dishes were incubated at the temperature of $37 \pm 0.5{ }^{\circ} \mathrm{C}$ for $24 \mathrm{~h}$ to allow the growth of microorganisms. The zone of inhibition produced by the microspheres loaded CTZ tablet towards the organism was measured $(\mathrm{mm})$.

\section{In vivo bioadhesive strength}

Three female rabbits, weighing between $2.6-3.2 \mathrm{~kg}$, were taken for the study. Ethical clearance for the handling of experimental animals was obtained from the institutional animal Ethical Committee (IAEC) of the JSS College of Pharmacy, Mysore, India. The animals were fasted for $12 \mathrm{~h}$ and optimized bioadhesive tablets were administered intravaginally. Pictures were taken using a 3-mm probe (laparoscopic probe with a 3-chip camera and Neosurge turn light source) (CXS-OP-SZL, Crystal Photonics, Germany) at different time intervals.

\section{Statistical data analysis}

Statistical data analysis was performed using the $t$-test. 


\section{RESULTS AND DISCUSSION}

In the present study, microspheres were prepared using various polymers. Spray drying method was optimized by using Eudragit RS-100, Eudragit RL-100 and a solvent (dichloromethane) to entrap the drug. Being a water insoluble drug, CTZ could be entrapped into water insoluble polymers by the spray drying method.

Polymers Eudragit RL 100 and RS 100 were selected for microsphere preparation because they are insoluble in aqueous media but are permeable, have the ability to produce $\mathrm{pH}$-independent drug-release profiles and have release rate controlling ability, non-toxicity, non-irritancy, stability at vaginal $\mathrm{pH}$ and compatibility with the drug (18). HPMC, SCMC, and Carbopol 934 were selected as polymers for tablet preparation because they have provided excellent bioadhesive properties on the mucosal surface and have been used in formulating various delivery systems (6).

The optimal inlet temperature for the preparation of CTZ microspheres was found to be $80^{\circ} \mathrm{C}$ while the optimal feed flow rate was $5-6 \mathrm{~mL} \mathrm{~min}^{-1}$. Increase in the feed flow rate resulted in particle size reduction and a decrease in the yield of microspheres. On the other hand, decrease in the feed flow rate below 5-6 mL min ${ }^{-1}$ increased the particle size, which was not suitable for pharmaceutical purposes. The solvent in the droplets could not be fully evaporated when the feed rate was $>5-6 \mathrm{~mL} \mathrm{~min}^{-1}$. It was observed that some of liquid droplets were attached inside the wall of the main chamber. Hence, an increase in feed rate led to higher solvent contents, especially at low inlet temperatures. Higher inlet temperature promoted a decrease in residual solvent by enhancing solvent evaporation.

It was observed that also compressed air flow and air pressure influenced the shape as well as the size distribution of microspheres, possibly because of variable shear force experienced by the particulate system. The average microsphere size increased with a decrease in spray flow. At higher feed rate and pressure, the system tended to produce irregularly shaped microspheres when it was not possible to distinguish between individual microspheres.

The flow property of microspheres was studied by calculating the angle of repose $(\theta$ in degrees) and compressibility index (CI, \%). The obtained data along with related parameters are presented in Table I. The values of $\theta$ ranged from 31.5 to $35.2^{\circ}$ indicating that the microspheres had good flow properties. The CI value was found to be in the range of 19.4 to $25.3 \%$, which also indicated good flow properties.

\section{FTIR studies}

The FTIR spectra of pure CTZ and microsphere formulation M4 are reported in Fig. 1. Positions of peaks in FTIR spectra of pure CTZ were compared with the spectrum of CTZ containing microspheres. Characteristic IR absorption peaks of pure CTZ of the aromatic $\mathrm{C}-\mathrm{H}$ stretch $\left(3167 \mathrm{~cm}^{-1}\right)$, aromatic $\mathrm{C}=\mathrm{C}$ stretch $\left(1589 \mathrm{~cm}^{-1}\right), \mathrm{C}=\mathrm{N}$ stretch $(1570$ $\left.\mathrm{cm}^{-1}\right)$ and aromatic $\mathrm{C}-\mathrm{H}$ bending $\left(761 \mathrm{~cm}^{-1}\right)$ were present in the spectrum of the CTZ tablet loaded with microspheres. Peaks at wavelengths corresponding to the pure drug were also observed in the microsphere formulation M4. The FTIR spectra of the pure drug and formulation indicated that the positions of characteristic peaks of CTZ were not altered after their successful entrapment in the microspheres, suggesting the absence of interactions between the drug and other components of the formulation (19). 


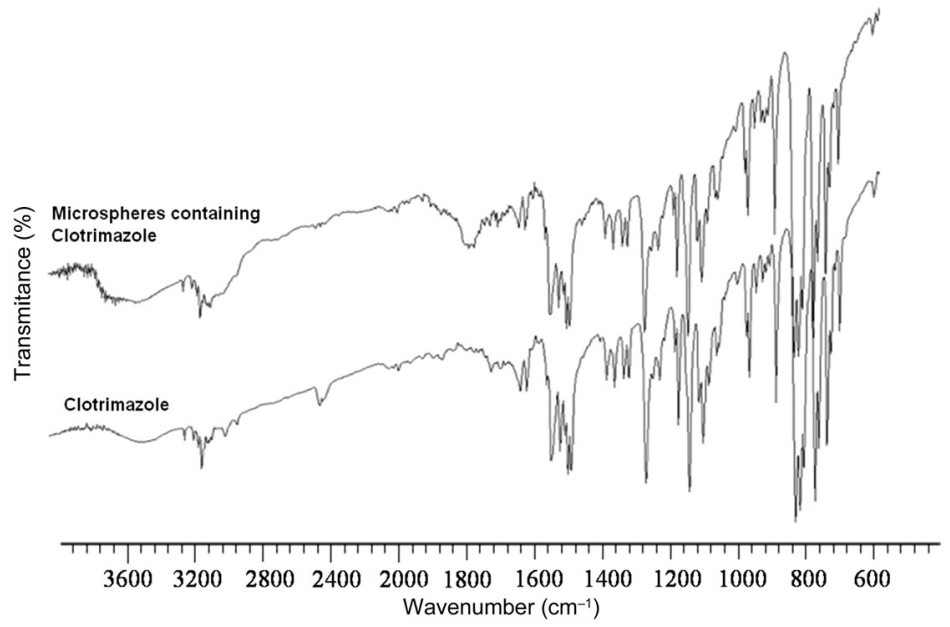

Fig. 1. FTIR spectra of pure drug clotrimazole and microspheres M4.

\section{Differential scanning calorimetry (DSC)}

DSC thermograms of pure drug and drug loaded microspheres (M4) are shown in Fig. 2. The DSC thermogram of pure CTZ showed a sharp melting endotherm at $144.47^{\circ} \mathrm{C}$. This melting endotherm was also observed for $\mathrm{M} 4$ at $144.23^{\circ} \mathrm{C}$, indicating the absence of

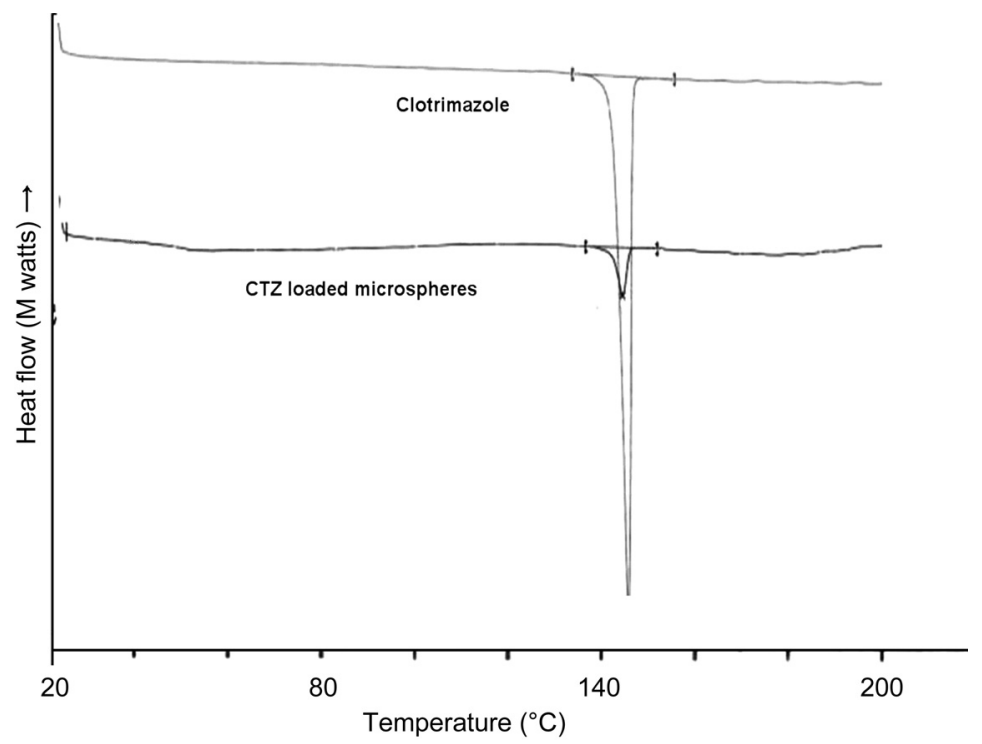

Fig. 2. DSC spectra of pure clotrimazole and microspheres M4. 
drug and polymer interactions. However, the melting endotherm of M4 was not as sharp as that of pure CTZ, which may be a result of the presence of polymers and the change in heat capacity of the polymer as it undergoes transition from the glassy to liquid state during microsphere formation (20).

\section{Particle size}

In general, the size of microspheres ranged from 17 to $58 \mu \mathrm{m}$. Particle size increased with an increase in polymer concentration and particle size decreased with a decrease in polymer concentration. For instance, as the amount of polymer increased from 1:1 to 1:3, the particle size increased by the same factor. This can be explained by the fact that at higher polymer concentration, the viscosity of polymer solution increased, thereby producing bigger droplets during emulsification, which were later hardened due to the evaporation of dichloromethane.

\section{SEM and sphericity}

SEM photographs of M2 and M4 are shown in Fig. 3. Non-aggregated microspheres with spherical shape were obtained for both formulations. Moreover, M4 showed a smooth surface, indicating that CTZ might be well dispersed inside the carrier. The sphericity factor was obtained in the range of 1.00 to 1.04 , indicating that the prepared formulations were spherical.
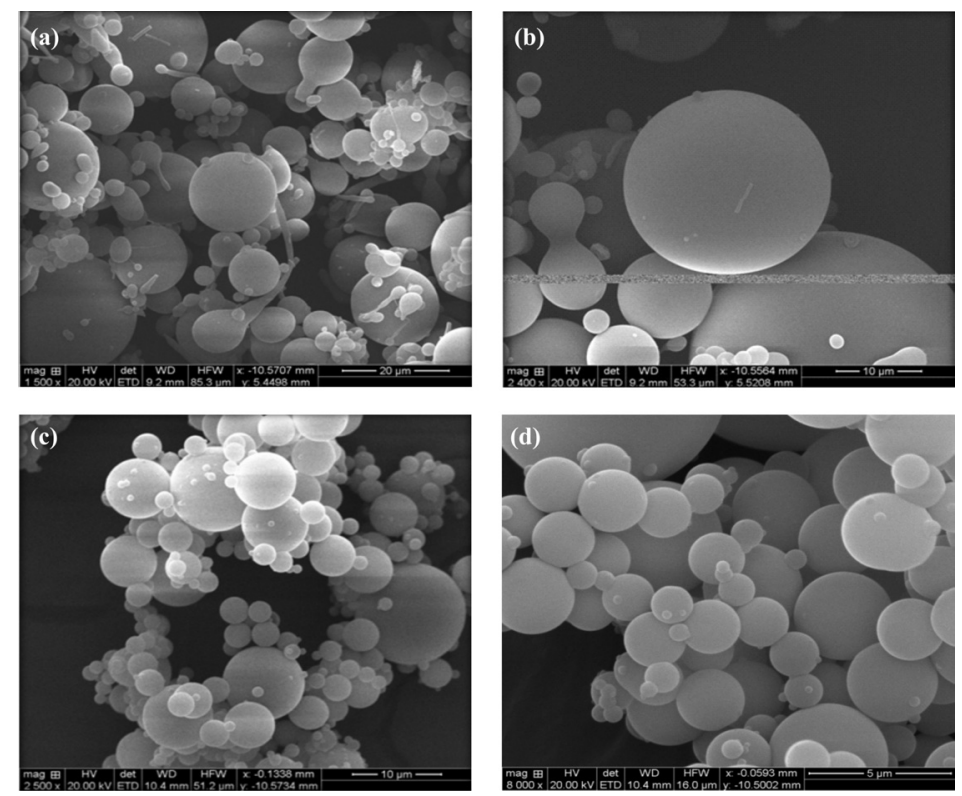

Fig. 3. SEM pictures of microsphere formulation M2 at: a) 1500× b) $2400 \times$ magnification, and M4 at: c) $2500 \times$ and d) $8000 \times$ magnification. 
N. Vishal Gupta et al.: Bioadhesive vaginal tablets containing spray dried microspheres loaded with clotrimazole for treatment of vaginal Candidiasis, Acta Pharm. 63 (2013) 359-372.

\section{Drug loading and encapsulation efficiency}

Encapsulation efficiency ranged from 55 to $88 \%$. It was found that the encapsulation efficiency and drug content increased with increasing amounts of polymers in the microspheres. Formulations M3 and M6 showed a relatively higher encapsulation efficiency, these formulations contained a high polymer concentration. It can be inferred from the results that there was a proper distribution of CTZ in the microspheres. The decrease in drug content in the product was probably due to the loss of drug with the exhaust gas during the spray drying process. During the microencapsulation process, mechanical variables cause loss of the final product and hence process yield may not be $100 \%$. Among all formulations, M1 containing $1000 \mathrm{mg}$ of Eudragit RS 100 and M4 containing $1000 \mathrm{mg}$ of Eudragit RL 100 showed maximum drug loading of 27.9 and $28.6 \%$, respectively. The results obtained are given in Table I.

Mucoadhesive tablets prepared were evaluated and the results obtained are given in Table II. Thickness, average mass, hardness and percentage friability of the prepared tablets were found to be satisfactory (14).

All the formulations showed considerable swelling properties in acidic $\mathrm{pH}$, which was due to the presence of hydrophilic polymers in the formulations. Polymeric matrices start to swell and build a gel layer around the tablet core when they come in contact with the medium which governs drug release. HPMC took more time to swell and was able to maintain the integrity of tablets. SCMC and Carbopol 934 are readily swellable polymers and showed good mucoadhesion properties. During the initial hours, swelling was the predominant factor compared to erosion. Percentage of swelling gradually decreased in all formulations after 10 hours, since the polymer eroded at a faster rate compared to the initial slow erosion. Results are shown graphically in Fig. 5. Similar results are reported in the literature (7).

\section{In vitro drug release studies}

The release profile of the drug from microspheres clearly indicates that the concentration of polymers slows the release of CTZ from microspheres. At the end of $12 \mathrm{~h}$, in vitro drug release from formulations M1 to M6 was found to be 83.1 to $98.9 \%$ in the vaginal environment, as shown in Fig. 4 . The total cumulative quantity of the drug released

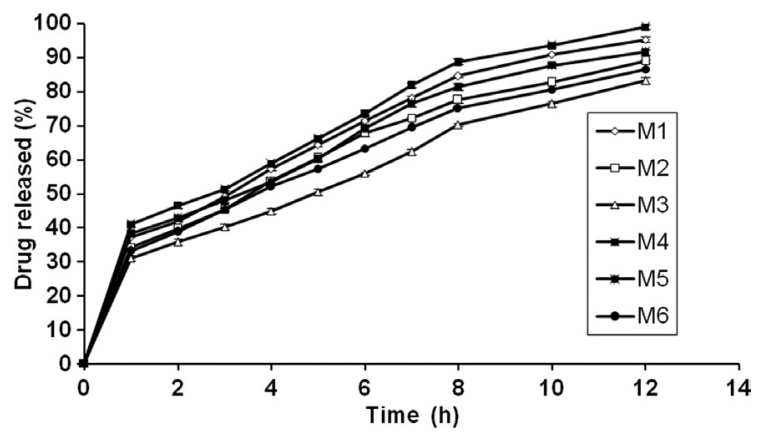

Fig. 4. Drug release profile of clotrimazole from microspheres (mean $\pm \mathrm{SD}, n=3$ ). 
at the end of the $12 \mathrm{~h}$ dissolution test was below $100 \%$ for all dosage forms. This may be in part due to the relatively slow erosion of the matrix under these test conditions, with a resultant slow release of entrapped drug from the matrices undergoing testing. Among various formulations, M4 was found to have a good release pattern and controlled release up to $12 \mathrm{~h}$. Hence, M4 was selected as the optimized formulation and incorporated in the prepared tablet.

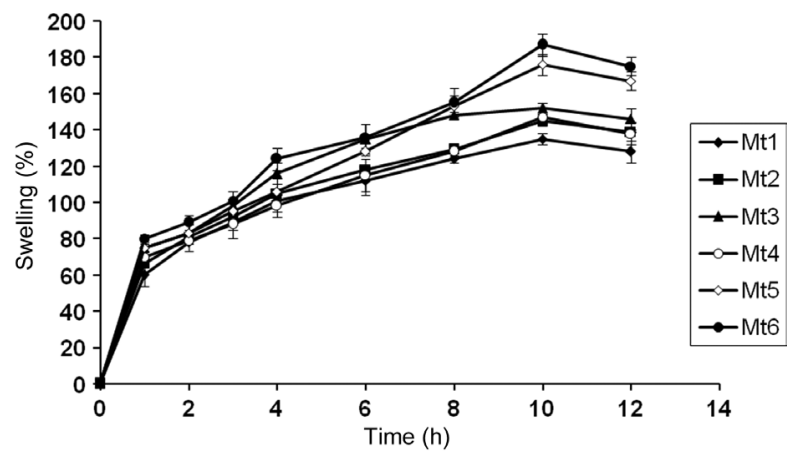

Fig. 5. Swelling of bioadhesive tablet formulations in citrate phosphate buffer $\mathrm{pH} 4.5$ (mean \pm SD, $n=3)$.

Formulations made with a single polymer like HPMC showed a burst effect when they came in contact with the fluid. To avoid such a burst effect, a combination of polymers was used in different ratios. The selected polymers such as HPMC, SCMC, and Carbopol 934 showed better mucoadhesive properties when they were used in higher concentrations and thereby released the drug for a prolonged period of time. It was found that the Mt6 formulation containing a combination of polymers HPMC and

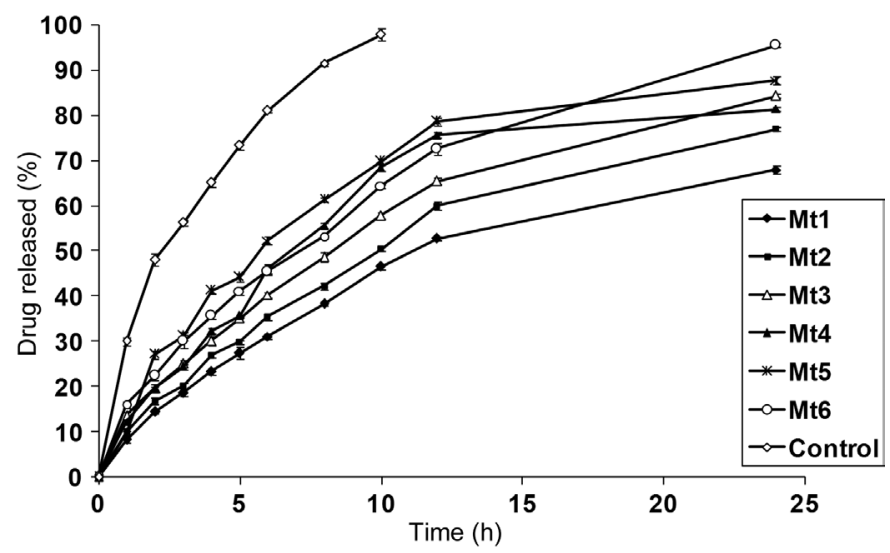

Fig. 6. Drug release profile of tablet formulations and control tablet (mean $\pm \mathrm{SD}, n=3$ ). 
Carbopol showed better in vitro release of $95 \%$ after $24 \mathrm{~h}$ as well as mucoadhesive properties. These results are in good agreement with the previous study (7). In vitro drug release was considerably retarded from the tablet compared to control tablets. From the data, Mt6 was chosen as the optimized formulation and compared with the control tablet. Control tablet released a maximum of $98 \%$ of CTZ up to $12 \mathrm{~h}$. Bioadhesive tablets provided controlled release of CTZ up to $24 \mathrm{~h}$. Drug release was faster from the control tablet in the initial period; about $65 \%$ of the drug was released after $5 \mathrm{~h}$ and $98 \%$ was released by the $10^{\text {th }}$ hour, whereas drug release from Mt6 was controlled up to $24 \mathrm{~h}$; about $72 \%$ of the drug was released after $12 \mathrm{~h}$ (Fig. 6). A previous report showed controlled release up to $6 \mathrm{~h}$ using chitosan-thioglycolic acid conjugate (8). In another work, drug release retarding agents were not employed, which resulted in controlled release of up to $6 \mathrm{~h}$ but the duration of bioadhesion was reported to be up to $50 \mathrm{~h}$.

\section{In vitro antifungal studies}

An antifungal study with Sabouraud culture medium showed that the CTZ tablet loaded with microspheres was able to control (inhibit) the growth of Candida albicans for more than $12 \mathrm{~h}$. The placebo tablet did not show any zone of inhibition. The Mt6 formulation showed an average zone of inhibition of $18.6 \pm 0.5 \mathrm{~mm}$, which was higher compared to the average zone of inhibition of pure metronidazole $(13.4 \pm 0.4 \mathrm{~mm})$.

\section{In vitro and in vivo bioadhesive strength}

In vitro mucoadhesion testing of tablets was done by detachment force measurements. Increased polymer concentration caused an increase in mucoadhesive strength. The combination of HPMC with Carbopol formulation showed higher mucoadhesive strength than the formulation with SCMC. The optimized bioadhesive formulation helped the tablet stay in the vagina and thereby enhanced its retention (Table II).

The bioadhesive tablet Mt6 was administered intravaginally to a rabbit and showed tablet bioadhesion and retention for $24 \mathrm{~h}$ (data not shown). It was evident that the tablet showed swelling, remained intact, and adhered to the vaginal mucous membrane for over $12 \mathrm{~h}$. After $24 \mathrm{~h}$, a gelous mass remaining on the spot of administration was seen. The study was in accord with the in vitro studies and confirmed the bioadhesion and retention of the tablet in the vagina.

\section{CONCLUSIONS}

The system of bioadhesive tablets containing CTZ loaded microspheres for bioadhesive vaginal drug delivery has demonstrated their effectiveness for the intended action. The in vitro bioadhesive strength and adhesion time studies have shown the ability of these tablets to adhere to the vaginal mucosa for an extended period of time as well as to improve drug availability. It can be concluded from the results of the present experimental work, that the formulation is easy to administer, simple and comfortable. Hence, clotrimazole could be formulated into this type of drug delivery system for controlled drug release. 
N. Vishal Gupta et al.: Bioadhesive vaginal tablets containing spray dried microspheres loaded with clotrimazole for treatment of vaginal Candidiasis, Acta Pharm. 63 (2013) 359-372

Acknowledgements. - The authors are grateful to Dr. H. G. Shivakumar, Principal, JSS College of Pharmacy, Mysore. The authors also thank the Strides Arco Lab Pvt. Ltd India for providing the necessary facility for obtaining DSC of the samples.

\section{REFERENCES}

1. A. H. F. Ahsan and A. Hussain, The vagina as a route for systemic drug delivery, J. Control. Release 103 (2005) 301-313; DOI: 10.1016/j.jconrel.2004.11.034.

2. H. Y. Karasulu, S. Hilmioglu, D. Y. Metin and T. Guneri, Efficacy of a new ketoconazole bioadhesive vaginal tablet on candida albicans, Farmaco 59 (2004) 163-167; DOI: 10.1016/j.farmac. 2003.11.018.

3. S. A. Sreenivas and K. V. Pai, Thiolated chitosans: Novel polymers for mucoadhesive drug delivery: A review, Trop. J. Pharm. Res. 7 (2008) 1077-1088.

4. C. Valenta, The use of mucoadhesive polymers in vaginal delivery, Adv. Drug Deliv. Rev. 57 (2005) 1692-1712; DOI: 10.1016/j.addr.2005.07.004.

5. S. Kawarkhe and S. S. Poddar, Designing of the mucoadhesive intravaginal spermicidal films, Indian J. Pharm. Sci. 72 (2010) 652-655; DOI: 10.4103/0250-474X.78540S.

6. S. Roy, K. Pal, A. Anis, K. Pramanik and B. Prabhakar, Polymers in mucoadhesive drug delivery system: A brief note, Design Monom. Polym. 12 (2009) 483-495; DOI: 10.1163/138577209X12478 283327236.

7. G. Sharma, S. Jain, A. K. Tiwary and G. Kaur, Once daily bioadhesive vaginal clotrimazole tablets: Design and evaluation, Acta Pharm. 56 (2006) 337-345.

8. C. E. Kast, C. Valenta, M. Leopold and A. Bernkop-Schnürch, Design and in vitro evaluation of a novel bioadhesive vaginal drug delivery system for clotrimazole, J. Control. Release 81 (2002) 347-354; DOI: 10.1016/S0168-3659(02)00077-9.

9. R. Vehring, W. R. Foss and L. David, Particle formation in spray drying, J. Aer. Sci. 38 (2007) 728-746; DOI: 10.1016/j.jaerosci.2007.04.005.

10. N. M. Ranjha, H. Khan and S. Naseem, Encapsulation and characterization of controlled release flurbiprofen loaded microspheres using bees wax as an encapsulating agent, J. Mater. Sci. Mater. Med. 21 (2010) 1621-1630; DOI: 10.1007/s10856-010-4034-4.

11. D. Perumal, C. M. Dangor, R. S. Alcock, N. Hurbons and K. R. Moopanar, Effect of formulation variables on in vitro drug release and micromeritic properties of modified release ibuprofen microspheres, J. Microencapsul. 16 (1996) 475-487.

12. A. A. Kahtani and B. S. Sherigara, Controlled release of theophylline through semi-interpenetrating network microspheres of chitosan-(dextran-g-acrylamide), J. Mater. Sci. Mater. Med. 20 (2009) 1437-1445.

13. C. R. Palem, R. Gannu, S. K. Yamsani, V. V. Yamsani and M. R. Yamsani, Development of bioadhesive buccal tablets for felodipine and pioglitazone in combined dosage form: in vitro, ex vivo, and in vivo characterization, Drug Deliv. 18 (2011) 344-352; DOI: 10.3109/10717544.2011. 557786 .

14. A. Madgulkar, S. Kadam and V. Pokharkar, Studies on formulation development of mucoadhesive sustained release itraconazole tablet using response surface methodology, AAPS PharmSci Tech. 9 (2008) 998-1005; DOI: 10.1208/s12249-008-9119-8.

15. United States Pharmacopoeia 30, National Formulary 25, USP Convention, Rockville (MD) 2007.

16. Y. M. Rao, Design and evaluation of mucoadhesive drug delivery systems, Indian Drugs 35 (1998) 558-565. 
N. Vishal Gupta et al.: Bioadhesive vaginal tablets containing spray dried microspheres loaded with clotrimazole for treatment of vaginal Candidiasis, Acta Pharm. 63 (2013) 359-372.

17. A. A. Dangi, N. R. Sheth, H. J. Patel, T. M. Shukla and H. M. Patel, Formulation and evaluation of once daily mucoadhesive vaginal tablet of clotrimazole using natural and synthetic polymers, Asian J. Pharm. Health Sci. 1 (2011) 176-182.

18. B. Nath, L. K. Nath and P. Kumar, Preparation and in vitro dissolution profile of zidovudine loaded microspheres made of Eudragit RS 100, RL 100 and their combinations, Acta Pol. Pharm. 68 (2011) 409-415.

19. S. Maiti, S. Kaity, S. Ray and B. Sa, Development and evaluation of xanthan gum-facilitated ethyl cellulose microsponges for controlled percutaneous delivery of diclofenac sodium, Acta Pharm. 61 (2011) 257-270; DOI: 10.2478/v10007-011-0022-6.

20. Y. Garg and K. Pathak, Design and in vitro performance evaluation of purified microparticles of pravastatin sodium for intestinal delivery, AAPS PharmSciTech. 12 (2011) 673-682; DOI: 10. 1208/s12249-011-9626-x. 